\title{
Übersichten
}

Zbl Arbeitsmed 2021 $\cdot 71: 192-199$ https://doi.org/10.1007/s40664-021-00424-y Eingegangen: 8. Oktober 2020 Überarbeitet: 28. Januar 2021 Angenommen: 31. Januar 2021 Online publiziert: 3. März 2021 (c) Der/die Autor(en) 2021

David H. Seidel ${ }^{1,2} \cdot$ Rolf P. Ellegast ${ }^{1} \cdot$ Monika A. Rieger ${ }^{2} \cdot$ Benjamin Steinhilber $^{2}$. Britta Weber $^{1}$

${ }^{1}$ Institut für Arbeitsschutz der Deutschen Gesetzlichen Unfallversicherung (IFA), Sankt Augustin, Deutschland

${ }^{2}$ Institut für Arbeitsmedizin, Sozialmedizin und Versorgungsforschung (IASV), Universitätsklinikum Tübingen, Tübingen, Deutschland

\section{Messdatenbasierte Gefährdungsbeurteilung}

\section{Kategorisierung messtechnischer Methoden zur Beurteilung physischer Belastungen der oberen Extremität}

Zur Verbesserung der Prävention

Bei Gefährdungsbeurteilungen physischer Belastungen kann zwischen beobachtungs- und messdatenbasierten Methoden unterschieden werden. Messdatenbasierte Verfahren zeichnen sich durch Objektivität und Genauigkeit aus und werden zunehmend kostengünstiger und praktikabler. Dieser Artikel gibt eine Übersicht zu potenziellen messtechnischen Verfahren und unterstützt betriebliche Akteure bei der Auswahl geeigneter Methoden für die jeweilige Bewertungssituation.

Die Durchführung der Gefährdungsbeurteilung physischer Belastungen ist ein zentraler Bestandteil deutscher Arbeitsschutzrichtlinien, Vorschriften, Gesetze und Rechtsverordnungen. Arbeitgeber sind verpflichtet (Arbeitsschutzgesetz [3], § 5 I-III), im Rahmen der Gefährdungsbeurteilung die relevanten Gefährdungen an Arbeitsplätzen, -bereichen oder bei auszuführenden Tätigkeiten zu erfassen, zu bewerten, Schutzmaßnahmen abzuleiten und deren Wirksamkeit zu überprüfen. Gefährdungsbeurteilungen tragen zur Prävention arbeitsbezogener Muskel-Skelett-Belastungen bei, was als ein wichtiges Ziel der Gemeinsamen Deutschen Arbeitsschutzstrategie (GDA) von Bund und Ländern sowie Trägern der gesetzlichen Unfallversicherung vereinbart wurde. und des Arbeitsschutzes macht die Europäische Agentur für Sicherheit und Gesundheitsschutz am Arbeitsplatz (EuWork, EU-OSHA) in der aktuellen Kampagne „Gesunde Arbeitsplätze - entlasten Dich!“ auf die Förderung von Präventionsmaßnahmen und Gefährdungsbeurteilungen in Verbindung mit arbeitsbezogenen Muskel-Skelett-Erkrankungen (MSE) aufmerksam [12]. Bis 2022 möchte die EU-OSHA eine Übersicht geeigneter Methoden und Bewertungsinstrumente bereitstellen.

Zwischen 20 und $57 \%$ aller arbeitsbezogenen MSE betreffen den Hand-ArmSchulter-Bereich [24]. Fehlbelastungen der oberen Extremitäten sind damit eine häufige Ursache für krankheitsbedingte Arbeitsausfälle. Für Gefährdungsbeurteilungen ist es erforderlich, entsprechende Risikofaktoren im Vorfeld zu identifizieren, um eine Auswahl geeigneter Expositionsbewertungsmethoden treffen zu können. Neben hoch repetitiver oder kraftvoller Arbeit sowie ungünstigen Haltungen wurden auch Kombinationen aus Kraftanforderungen und dem Bewegungsverhalten als arbeitsbedingte Risikofaktoren der oberen Extremität bereits quantitativ beschrieben [25].

Für Gefährdungsbeurteilungen physischer Belastungen wurde bereits 2010 ein 5-stufiges Ebenenmodell vorgeschlaropean Agency for Safety and Health at gen [11], das die Grundlage für das Vorgehen im Projekt „Mehrstufige Gefährdungsanalyse physischer Belastungen am Arbeitsplatz" (MEGAPHYS) $[6,9]$ darstellte sowie im Bereich der oberen Extremität angewandt werden kann. Die Ebenen lassen sich in beobachtungsbasierte (Grob-Screening, Spezielles Screening, Experten-Screening) und messtechnische Verfahren (betriebliche Messung, Labormessungen/Simulationen) einteilen.

Der Vorteil von beobachtungsbasierten Methoden liegt vor allem in der einfachen und praxisnahen Anwendung, insbesondere bei zusätzlicher Beurteilung von Arbeitsplatzumgebungen, -bedingungen oder -organisation $[6,9]$. Sie sind vorteilhaft für erste Expositionsabschätzungen. In der Literatur werden jedoch auch einige Limitationen beschrieben. Beobachtungsbasierte Verfahren können durch subjektive Erfahrungen beeinflusst werden [13]. Das heißt, zwischen unterschiedlichen Beobachtenden kann es zum Teil zu erheblichen Unterschieden bei der Bewertung kommen. Weitere Einflussfaktoren sind z. B. Blickwinkel (Verdeckung), Tagesform oder Erinnerungsvermögen [13, $16,17,23$ ]. Holtermann et al. [17] merken in diesem Zusammenhang ebenfalls an, dass erfahrene Beobachter benötigt werden, was kostenintensiv für jede beobachtete Arbeitszeiteinheit ist und meist kurze Beurteilungssequenzen oder 
limitierte Stichprobenanzahlen zur Folge hat. Beobachtungen können auch zu ethischen Komplikationen führen, z.B. bei Tätigkeiten in der Pflege [17]. Beobachtungsverfahren scheinen für grobe Erhebungen ausreichend zu sein, jedoch sind für weitere Untersuchungen ergänzende technische Methoden mit höherer Reliabilität von Vorteil [13, 23].

Lin et al. [18] weisen bspw. darauf hin, dass messwertbasierte Systeme in den letzten Jahren praktikabler und genauer geworden sind, längere $\mathrm{Be}$ triebszeiten ermöglichen und in der Lage sind, mehr Daten als früher zu speichern bzw. zu verarbeiten. Diese Verfahren sind objektiv, weisen einen hohen Detaillierungsgrad auf und ermöglichen eine genaue Quantifizierung von Expositionen. Ebenfalls werden technische Methoden für die Erstellung objektiver Expositionskataster [10] und für Analysen komplexer Arbeitsplätze mit schnell wechselnden oder parallel vorkommenden Belastungsarten [15] eingesetzt. Auch für Risikoabschätzungen zur Evaluation von Interventionen werden messtechnische Methoden empfohlen [23]. Messtechnische Methoden zur Erfassung der physischen Arbeitsbelastung unterliegen keinen subjektiven Verzerrungen, sind in der Regel auch an verdeckten oder engen Arbeitsplätzen anwendbar und weisen eine hohe Validität und Reliabilität auf [16]. Limitationen waren bisher ein gegenüber Beobachtungsverfahren höherer Zeitund Kostenaufwand (oftmals bedingt durch aufwendige Instrumentierungen und Auswertungen), eine Nutzung vorrangig durch Experten und eine mögliche Interferenz mit dem Arbeitsablauf [14]. Durch den Technikfortschritt werden jedoch die objektiven und genauen Systeme immer praktikabler und günstiger $[13,18]$ und bereits international zur Gefährdungsbeurteilung empfohlen [16]. Das bietet gute Voraussetzungen für andere Nutzergruppen und künftige Weiter- und Neuentwicklungen messwertgestützter Methoden bzw. Bewertungsverfahren auch im Rahmen der hierzulande durchzuführenden Gefährdungsbeurteilung physischer Belastungen, z. B. im Bereich der oberen Extremität. Bisher war es in der be- trieblichen Praxis sehr schwierig, die geeignete messtechnische Methode für die jeweiligen Zwecke auszuwählen.

Das Ziel dieser Arbeit ist es daher, eine übersichtliche Kategorisierung am Beispiel aktuell verfügbarer Methoden für die messtechnisch basierte Gefährdungsbeurteilung arbeitsbedingter physischer Belastungen im Bereich der oberen Extremität zu erstellen. Beispiele sollen die Einsatzbereiche verdeutlichen und betriebliche Praktiker zukünftig bei der Auswahl geeigneter messtechnischer Verfahren unterstützen.

\section{Messmethoden zur Belastungs- quantifizierung}

Zur objektiven Quantifizierung arbeitsbezogener Muskel-Skelett-Belastungen der oberen Extremität sind zahlreiche Methoden verfügbar. Kamerabasierte Methoden als Basis für automatisierte Bewegungsanalysen unter Verwendung von passiven oder aktiven, reflektierenden Markern sind überwiegend an spezielle stationäre Laborumgebungen gebunden. Entsprechend sind solche Methoden nur eingeschränkt für den mobilen Einsatz geeignet und somit in Abhängigkeit von den Rahmenbedingungen wenig praktikabel für eine praxisnahe Gefährdungsbeurteilung vor Ort, z.B. an nichtstationären Arbeitsplätzen. Für Quantifizierungen werden heutzutage auch videobasierte Mustererkennungen (nicht markerbasiert) verbunden mit biomechanischen Modellierungen verwendet [1]. Wenn die Arbeitsabläufe und Arbeitsinhalte dies erlauben, lassen sich videobasierte Verfahren oftmals problemlos in der Praxis an industriellen Arbeitsplätzen einsetzen, da als Hardware nur eine Videokamera, ein Smartphone oder Tablet benötigt wird. Jedoch ist hier zur Bewertung der Arbeitsplatzbelastungen eine spezifische zusätzliche Videoanalyse-Software notwendig. Zudem sind die bisher verfügbaren Bewertungsansätze häufig nur auf bestimmte Bewegungsmuster bezogen. Obwohl eine Datenerfassung prinzipiell möglich ist, erfordert die nachfolgende Auswertung somit erhöhte Aufwände, wie z.B. einen zusätzlichen Programmieraufwand. Problematisch ist ein Ka- meraeinsatz auch an Arbeitsplätzen, an denen aus datenschutzrechtlichen, ethischen oder betrieblichen Gründen keine Videoaufzeichnungen erfolgen dürfen. Nicht nur für derartige Arbeitsplätze, sondern auch für den Einsatz an nichtstationären Arbeitsplätzen eignen sich daher personengetragene Bewegungssensoren. Die Bandbreite erstreckt sich dabei vom Einsatz einzelner Beschleunigungssensoren bis hin zur Anwendung von Inertialsensoren als Multisensorsysteme, synchronisiert mit weiterer Sensorik, z. B. zur Erfassung von Kräften oder physiologischer Vorgänge.

\section{Kategoriensystem}

In der Literatur werden messtechnische Verfahren zur Erfassung und Bewertung beruflicher körperlicher Aktivität und spezifischer physischer Arbeitsbelastungen in einem Kategoriensystem eingeteilt, das eine Differenzierung in 3 Messsystem-Kategorien vorsieht [8, 16, 17, 29]. Im Folgenden wird dieses Kategoriensystem als Grundlage genutzt, um eine entsprechende Klassifizierung messtechnischer Systeme zur Erfassung und Bewertung arbeitsbezogener Belastungen der oberen Extremitäten vorzunehmen.

\section{Kategorie 1}

Hierzu zählen Messverfahren mit 1-2 Sensoreinheiten, welche die Belastung einer spezifischen Lokalisation (z. B. Handgelenk, Ellenbogen) abbilden. Solche Verfahren basieren in der Regel auf dem Einsatz von Bewegungs-, Haltungsoder Positionssensoren wie Accelerometer (Beschleunigungsmesser) oder Goniometer (Winkelmesser). Diese Sensoren waren früher oft kabelgebunden und kostenintensiv und ihre Handhabung setzte spezielle Kenntnisse für die Sensoranbringung und Datenauswertung voraus. Durch technische Optimierung sind die zur Erfassung von Arbeitsbewegungen geeigneten Systeme heute kostengünstiger und nutzerfreundlicher. Sie sind beispielsweise häufig kabellos, und durch intelligente Algorithmen werden Fehler bei der Anbringung oder Datenauswertung vermieden [29]. 


\section{Kategorie 2}

Mit Messverfahren mit $\geq 2$ Sensoreinheiten lassen sich Belastungen eines Lokalisationsbereichs betrachten (Kette von Lokalisationen, z.B. SchulterEllenbogen-Hand-Bereich). Die Sensoreinheiten können in smart textiles eingearbeitet oder individuell am Körper angebracht werden. Neben Sensorik zur Bewegungserfassung (z.B. Inertialsensoren) kann beispielsweise Elektromyographie (EMG), Nahinfrarotspektroskopie (NIRS) oder HandArm-Vibration(HAV)-Erfassungssensorik zum Einsatz kommen. Es gibt eine Reihe an wearables, die anhand von Inertialsensoren, Dynamometern und Oberflächen-EMG zur biomechanischen Datenerfassung bei der Arbeit als Basis für die Risikobewertung geeignet sind [22].

\section{Kategorie 3}

Bei diesen komplexen Messverfahren werden viele Sensoren kombiniert, um die Belastung mehrerer Ketten von Lokalisationen oder des gesamten Körpers zu betrachten. Hierzu zählen Multisensorsysteme, die in der Regel auf Inertialsensoren basieren, aber auch mit weiterer Messtechnik kombinierbar sind (z.B. Computer-unterstützte Erfassung und Langzeit-Analyse von Belastungen des Muskel-Skelett-Systems (CUELA), Xsens, [28]).

\section{Methodenüberblick und Anwendungsbeispiele}

Eine prinzipielle Übersicht zu den 3 Kategorien von Messsystemen ist in - Abb. 1 illustriert. Beispiele für die jeweilige Messsystemkategorie für die Erfassung und Bewertung arbeitsbezogener Belastungen der oberen Extremitäten sind in - Abb. 2 zusammengefasst. Hierbei erscheinen wegen der hohen Praktikabilität die Kategorien 1 und 2 für den Einsatz im Betrieb besonders bedeutsam. Beispiele für derartige Sensorik und mögliche Bewertungsansätze, die aus der Literatur herangezogen werden können, werden in $\bullet \mathbf{A b b} .3$ präsentiert.

Zbl Arbeitsmed 2021·71:192-199 https://doi.org/10.1007/s40664-021-00424-y

(c) Der/die Autor(en) 2021

D. H. Seidel · R. P. Ellegast · M. A. Rieger · B. Steinhilber · B. Weber

\section{Messdatenbasierte Gefährdungsbeurteilung. Kategorisierung messtechnischer Methoden zur Beurteilung physischer Belastungen der oberen Extremität}

\section{Zusammenfassung}

Hintergrund. Beobachtungsbasierte Methoden zur Gefährdungsbeurteilung physischer Belastungen im Bereich der oberen Extremität können durch subjektive Erfahrungen der Untersuchenden beeinflusst werden. Darüber hinaus ist eine Quantifizierung biomechanischer Belastungen, wie Zeitverläufe von Gelenkwinkeln, Winkelgeschwindigkeiten oder Kräften durch Beobachtungen, schwer möglich. Zur objektiven Quantifizierung von Expositionen im Rahmen von spezifischen Gefährdungsbeurteilungen eignen sich daher vor allem messtechnische Methoden, wobei die Auswahl der entsprechenden Methode herausfordernd sein kann.

Zielsetzung. Dieser Artikel soll Arbeitsschutzfachleute dabei unterstützen, aus der Bandbreite der unterschiedlichen Verfahren die geeignete messtechnische Methode für eine vorliegende Expositionssituation zu identifizieren.

Methoden. Ausgehend von einer Literaturübersicht wurden Messmethoden für die obere Extremität in Anlehnung an ein etabliertes Kategoriensystem hinsichtlich ihrer Komplexität klassifiziert. Zusätzlich werden Anwendungsbeispiele für alle Kategorien skizziert.

Ergebnisse. Dieser Artikel liefert eine Übersicht und Klassifizierung von unterschiedlichen messtechnischen Erfassungsund Bewertungsmethoden arbeitsbezogener Muskel-Skelett-Belastungen, die in 3 Kategorien von einfach bis komplex eingeteilt werden.

Diskussion. Vereinfachte Sensorik in Kombination mit spezifischen Bewertungsansätzen kann zukünftig die objektive Gefährdungsbeurteilung physischer Belastungen unterstützen.

Schlüsselwörter

Quantifizierung arbeitsbedingter Exposition . Objektives Bewertungsverfahren - Messsystem-Kategorie · Sensor · Schulter-EllenbogenHand-Bereich

\section{Measurement-based risk assessment. Categorization of measurement methods for assessing physical workloads of the upper extremity}

\section{Abstract}

Background. Observation-based methods for risk assessment of physical workloads of the upper extremity can be influenced by subjective experiences of the investigators. In addition, it is difficult to quantify biomechanical workloads, of e.g., time courses of joint angles, angular velocities, or forces by observations. For objective and precise quantification of exposures in the context of specific risk assessments, technical measurement-based methods are therefore particularly suitable, although the choice of the appropriate method can be challenging. Objective. This article is intended to support occupational safety experts to identify the appropriate measurement-based method for an existing exposure situation from the range of different methods.

Methods. Based on a literature review, measurement methods for the upper extremity were classified according to their complexity based on an established category system. In addition, application examples are presented for all categories.

Results. This article provides an overview and classification of different recording and assessment methods of work-related musculoskeletal loads, which are divided into three categories from simple to complex. Discussion. Simplified sensor technology in combination with specific assessment approaches might support the objective risk assessment of physical workload in the future.

\section{Keywords}

Quantification of work-related exposure · Objective assessment approach - Measurement system category - Sensor - Shoulder-ElbowHand-area 


\section{Kategorie 1}

Erfassung und Analyse von Expositionsparametern einer Lokalisation z. B. Haltung und Bewegung des Oberarms

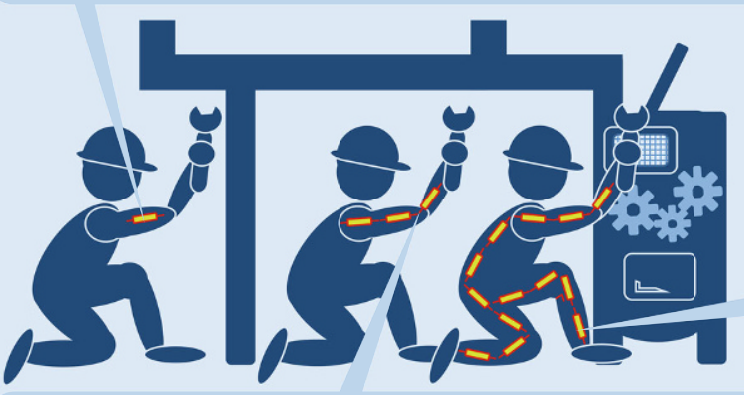

\section{Kategorie 3}

Erfassung und Analyse von Expositionsparametern mehrerer Lokalisationsbereiche

z. B. Bewegung des ganzen Körpers + Aktionskraft der Hände

Abb. $1<$ Kategorisierung von Messsystemen zur Erfassung und Bewertung arbeitsbezogener Belastungen der oberen Extremitäten in Anlehnung an die in der Literatur beschriebenen Klassifizierungen von Messungen physischer Aktivitäten. (Nach [8, 16, 17 ,

\section{z. B. Haltung und Bewegung des Schulter-Ellenbogen-Hand-Bereichs + Muskelaktivität des Unterarms}

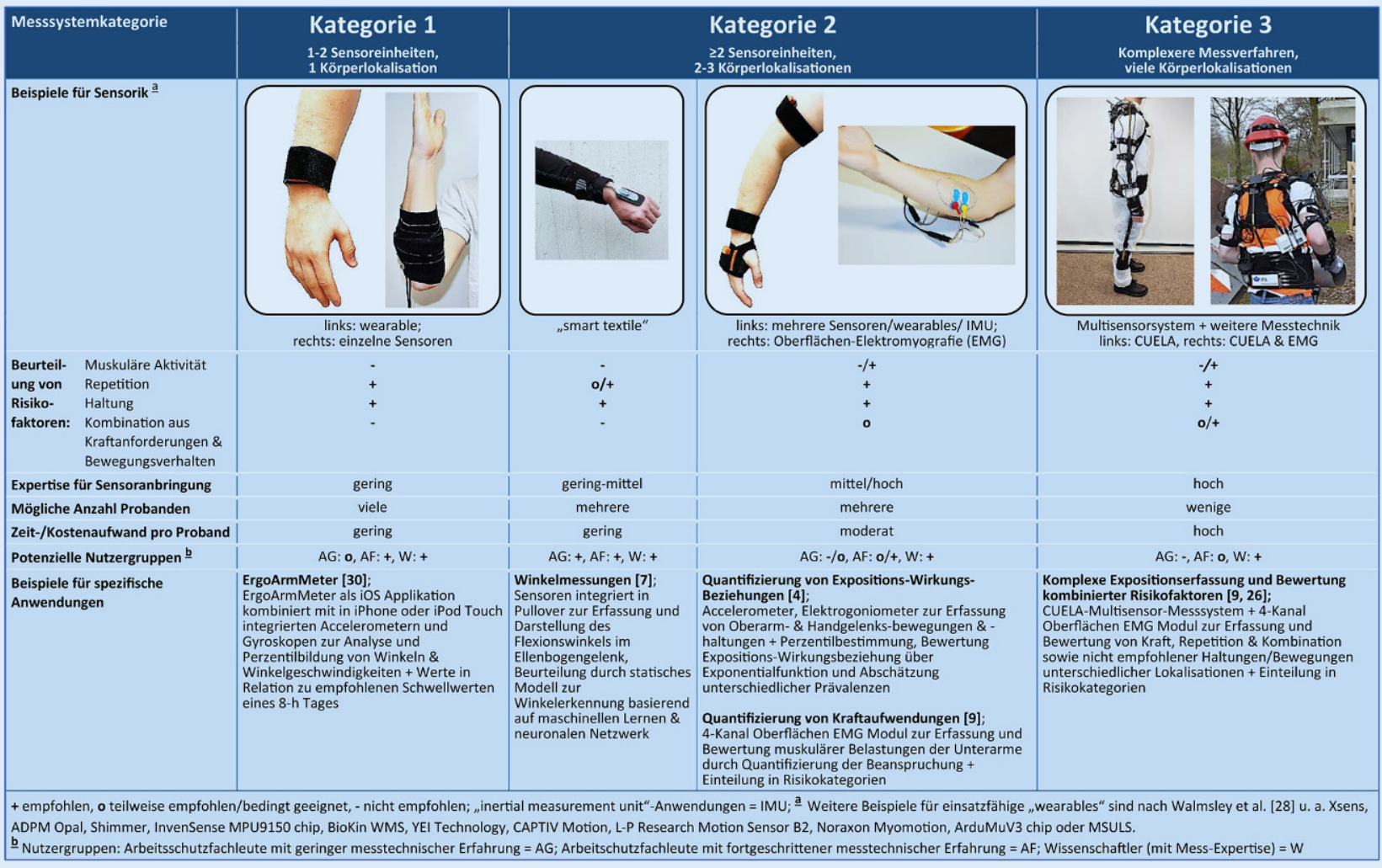

Abb. $2 \Delta$ Beispiele für mobile, körpergetragene messtechnische Methoden (Kategorien 1-3) zur Expositionsermittlung der oberen Extremität. Einschätzung bezüglich der erforderlichen Expertise für die Anbringung von Sensoren, der möglichen Anzahl von Probanden, des Zeit-/Kostenaufwands pro Proband und die Empfehlung bezüglich der Nutzergruppen basieren auf der PEROSH-Klassifikation (Partnership for European Research in Occupational Safety and Health, PEROSH). (Nach [4, 7, 9, $16,17,26,28,29,30])$ 


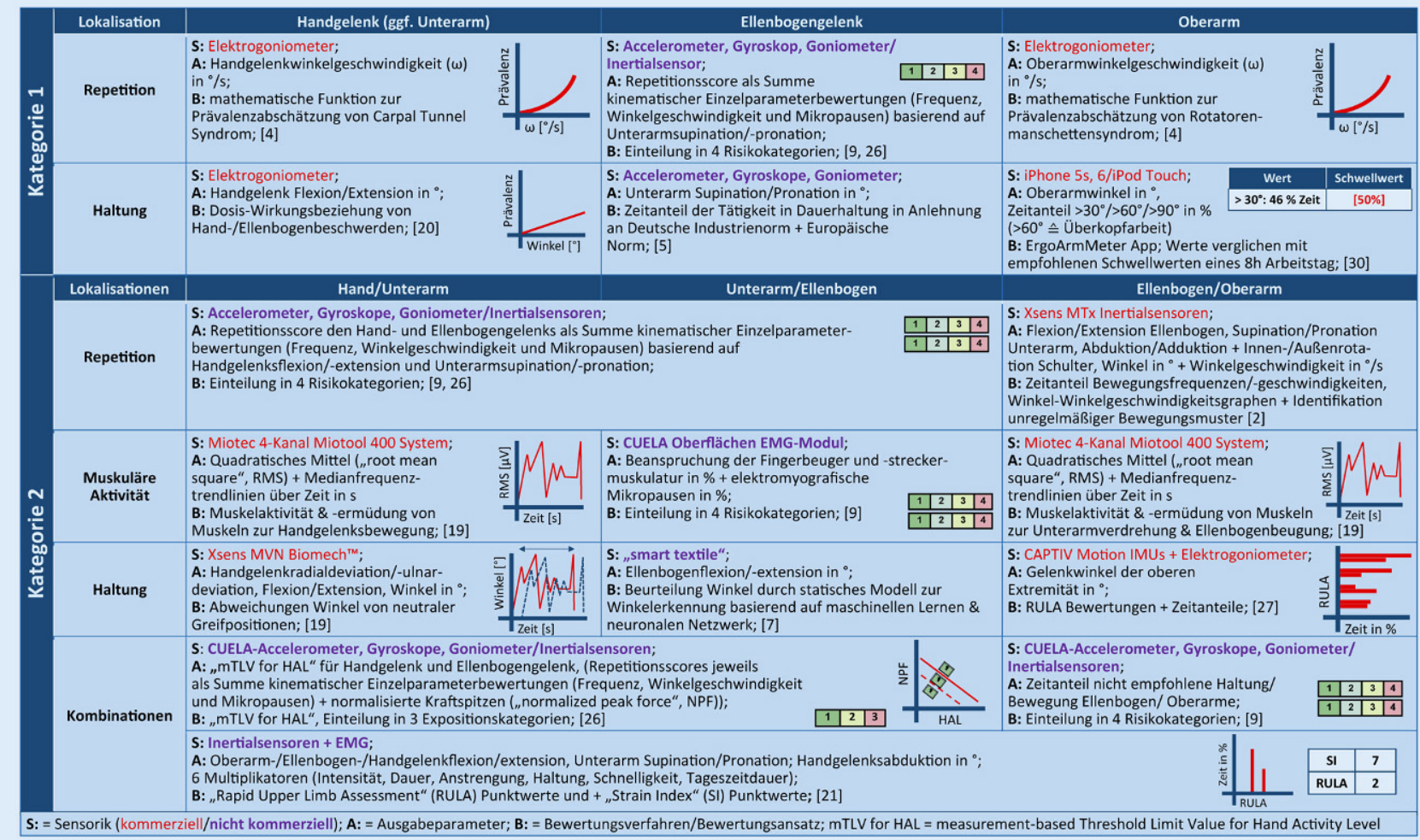

Abb. $3 \Delta$ Beispiele für Messtechnik, Ausgabeparameter und zugrundeliegende Bewertungsansätze für Geräte der Messsystemkategorien 1 und 2. (Nach $[2,4,5,7,9,19-21,26,27,30])$

\section{Anwendungsszenarien aus der betrieblichen Praxis}

Szenario a) Nach Umstrukturierungen in der Montagelinie treten bei Monteuren von Heckklappenkabelbäumen häufig Beschwerden im Schulterbereich auf. Da die Kabelbäume vorrangig über Schulterniveau montiert werden, entstehen Körperzwangshaltungen und problematische Gelenkbelastungen. Der Ergonomieexperte des Unternehmens wird beauftragt, besonders hohe Schulterbelastungen während der Montage $\mathrm{zu}$ identifizieren, um mögliche Verbesserungsmaßnahmen im Arbeitsprozess entwickeln zu können. Da nach der Tätigkeitsbeobachtung und orientierenden Bewertung ungünstige Oberarmhaltungen (z.B. Armhebungen über $60^{\circ}$ ) als Ursache für die Fehlbelastungen im Bereich der Schulter angenommen werden, eignet sich in diesem Fall zur Quantifizierung der Belastung ein einfaches System der Kategorie 1, wie z. B. der Einsatz eines Smartphones in Kombination mit einer entsprechenden Applikation (• Abb. 3,
Kategorie 1, Haltung, Oberarm). Die kostengünstige Applikation stellt unabhängig von Arbeitsplätzen und Teiltätigkeiten objektive Parameter bereit, wie z.B. Perzentile von Winkelverteilungen, prozentuale Schichtanteile ungünstiger Armhaltungen oder den Median der Winkelgeschwindigkeit, welche sich nicht genau durch Beobachtungen quantifizieren lassen. Basierend auf den Daten zur kumulativen Dauer von Armhebungen über $60^{\circ}$ sowie zur Dauer von ununterbrochenen Armhebungen über $60^{\circ}$ während einer typischen Arbeitsschicht lassen sich Belastungsspitzen genauer als durch die Beobachtung von umschriebener Dauer identifizieren. Durch die objektiven Parameter und den Vergleich von Winkeldaten mit empfohlenen Schichtschwellwerten sind Maßnahmen zur Reduktion der durchschnittlichen Winkelgeschwindigkeit denkbar, welche sich durch Beobachtungen nicht objektiv evaluieren lassen. Anhand des Schichtbelastungsprofils können dann gegebenenfalls Maßnahmen zur Reduzierung der Schulterbelastung abgeleitet werden, beispielsweise durch Job- bzw. Arbeitsplatzrotationen.

Szenario b) In einer großen Filiale im Lebensmitteleinzelhandel berichten die Beschäftigten von Beschwerden im Bereich der Handgelenke und Ellenbogen, vor allem bei Kassiertätigkeiten, weniger jedoch bei dem Auffüllen der Regale mit neuen Waren. Ausgehend von der Tätigkeitsbeobachtung wird vermutet, dass hochfrequente Beugungen der Handgelenke und Ellenbogen sowie Verdrehungen der Unterarme kombiniert mit den Warengewichten bei Tätigkeiten am Kassenband zu typischen Beschwerden und Erkrankungen im erweiterten Lokalisationsbereich (Handgelenk/ Ellenbogen) führen können. Um den Belastungsunterschied zwischen beiden Arbeitsplätzen zu quantifizierten, sollen jeweils die Belastungen im Hand-ArmBereich durch eine Arbeitsschutzfachkraft verglichen werden. Zur einfachen Handhabung eignet sich beispielsweise ein mit Inertialsensoren bestücktes smart textile aus Kategorie 2 zusätzlich 
$\mathrm{zu}$ einer handelsüblichen Waage für die Erfassung der Warengewichte. Die ermittelten Ergebnisse wie Winkel-Zeitverläufe, Anzahl und Gewichte bewegter Waren und die Bewertung des Ausmaßes der Repetition vor dem Hintergrund von Daten aus der Literatur können genutzt werden, um objektive Belastungsprofile $\mathrm{zu}$ erstellen. Diese Ergebnisse können die Basis für fundierte Anpassungen des Kassentisches sein, um ergonomische Tätigkeiten zu ermöglichen.

Szenario c) In einem Unternehmen, das sich auf Wegebau mit Betonsteinpflaster spezialisiert hat, steht die Geschäftsführung vor der Überlegung, eine neue kostenintensive Maschine anzuschaffen, welche die manuelle Steinsetzung in großen Teilen ersetzen soll. Die Geschäftsführung erhofft sich von der Maschine einen wirtschaftlichen Vorteil aufgrund der Zeitersparnis und der Reduzierung krankheitsbedingter Arbeitsausfälle. Die Beschäftigten hatten häufiger über Beschwerden in mehreren Lokalisationen (Arme, Schultern, Nacken, Rücken, Knie) geklagt. Daher ist in Kooperation mit einer Hochschule ein wissenschaftliches Projekt zum Vergleich des Pflasterns mit und ohne maschinelle Unterstützung geplant. Unter Berücksichtigung des Zeitfaktors sollen für beide Arbeitsweisen die Auswirkungen auf das Muskel-Skelett-System genau quantifiziert werden. Da die erwarteten Effekte auf den Bewegungsablauf komplex sind und den gesamten Körper betreffen, müssen die Genauigkeit und der Detaillierungsgrad der Ausgabeparameter sehr hoch sein. Daher ist für dieses Projekt ein Multisensorsystem der Kategorie 3 mit Oberflächen-EMG und HAV-Erfassungssensorik und mehreren hinterlegten Bewertungsansätzen zur Belastungsbeurteilung bei mehreren Lokalisationen geeignet. Durch die umfangreiche Messtechnik lassen sich komplexe Bewegungen, Haltungen, Kraftaufwendungen und mögliche Belastungen durch maschinenbedingte Schwingungen quantifizieren. Anhand der ermittelten Expositionsdaten beim Pflastern mit und ohne maschinelle Unterstützung lassen sich jeweils Schichtbelastungsprofile erstellen, in welchen beispielsweise Belastungen der Bandscheiben, ungünstige Körperhaltungen und Belastungsspitzen durch Repetitionen und hohe Kräfte enthalten sein können. Der ermittelte Expositionsunterschied soll in Kombination mit der zeitlichen Betrachtung bewertet werden und kann die Grundlage für die Kaufentscheidung liefern.

\section{Diskussion und Ausblick}

Die Methodenübersicht und Anwendungsbeispiele im vorliegenden Artikel sollen betrieblichen Praktikern einen aktuellen Einblick in messtechnische Erfassungsmethoden sowie die zur Bewertung der Belastungen herangezogenen möglichen Parameter aus der Literatur ermöglichen. Eine in der Literatur empfohlene Messsystemkategorisierung zur Erfassung und Bewertung physischer Aktivitäten $[8,16,17,29]$ wurde erweitert auf den Bereich der oberen Extremität angewandt. Dieses Vorgehen und die erarbeitete Übersicht bieten eine Orientierung für die Einsatzmöglichkeiten einer messdatenbasierten Gefährdungsbeurteilung arbeitsbezogener physischer Belastungen der oberen Extremität. Die erarbeiteten Empfehlungen im Hinblick auf unterschiedliche Nutzergruppen können darüber hinaus als Hilfestellung dienen, welche Art von Messtechnik für die jeweilige Beurteilungssituation am ehesten geeignet sein könnte.

Mit zunehmender Komplexität der Messsysteme steigen die Anforderungen an die erforderliche Expertise für die Anbringung von Sensoren und die Datenanalyse und somit der Zeit- und Kostenaufwand. Auch der Datenschutz ist bei der Expositionserfassung mit wearable technology einzuhalten. Neben der Weiterentwicklung von Bewertungsverfahren ist auch die Festlegung und Bereitstellung praktikabler kommerzieller Sensorik bzw. entsprechender Messsysteme wünschenswert. In diesem Zusammenhang ist auch die gezielte messtechnische Analyse einzelner Parameter und ausgewählter Lokalisationen denkbar. Durch ein spezifischeres Set-up und einfach zu bedienende Messtechnik wäre zukünftig eine deutliche Reduzierung von Aufwand und Komplexität bei betrieblichen Messungen möglich. Software gestützt sind so auch schnellere und objektive Auswertungen möglich, wobei zielstellungsabhängig die Analysen problemlos erneut durchgeführt oder erweitert werden können. Neben Wissenschaftlern könnten auch betriebliche Praktiker Messdaten erheben. Um mögliche Fehlinterpretationen von Daten und damit verbundene fehlerhafte Gefährdungsbeurteilungen zu vermeiden, wird prinzipiell empfohlen sich über Möglichkeiten und Grenzen der jeweiligen Methode genau zu informieren. Dies gilt allerdings nicht nur für Messverfahren, sondern auch für alle anderen Verfahren der Gefährdungsbeurteilung.

Zur Expositionserfassung und -bewertung sind für die Lokalisationen Hand/Oberarm (Repetition, Haltung), Hand/Unterarm (Kraft, Haltung) und Ellenbogen/Oberarm (Repetition, Kraft, Haltung) bereits kommerziell verfügbare Sensoren mit Ausgabeparametern und Bewertungsansätzen als Grundlage für den Einsatz in der messdatenbasierten Gefährdungsbeurteilung physischer Belastungen vorhanden (• Abb. 3). Perspektivisch ist es jedoch empfehlenswert, dass Bewertungsverfahren, die aktuell auf Daten zurückgreifen, die mit nicht kommerziell verfügbarer Sensorik erhoben werden, weiterentwickelt und auf die Verwendung von Messdaten aus der Anwendung kommerziell verfügbarer Sensorik übertragen werden. Walmsley et al. [28] stellen dazu z.B. 13 kommerzielle wearable Sensoren vor, welche sich für eine entsprechende Übertragung eignen. Damit werden beispielsweise für MEGAPHYS entwickelte Bewertungen (z.B. Repetitionsscore (RepScore) [9, 26]) und darauf aufbauende Ansätze (z.B. measurement-based Threshold Limit Value for Hand Activity Level (mTLV for HAL) [26]) für betriebliche Praktiker verfügbar und können die messdatenbasierte Gefährdungsbeurteilung physischer Belastungen unterstützen. Die im Projekt MEGAPHYS entwickelten und validierten Bewertungsverfahren für messtechnische Analysen arbeitsbezogener Muskel-Skelett-Belastungen können als Grundlage für die Bewertung lokalisationsbezogener Belastungen mittels Messsystemen der Kategorien 1-3 
genutzt werden. Schnittstellen zu entsprechender kommerzieller Messtechnik werden derzeit am IFA definiert und implementiert, um den Zugang zu der Methodik für betriebliche Praktiker zu erleichtern.

Vor diesem Hintergrund empfiehlt sich die Anpassung des Ebenenmodells der Gefährdungsbeurteilung aus dem Jahr 2010 [11]. Dabei könnten Kategorie-1-Systeme durch eine einfache Handhabung das Spezielle Screening ergänzen. Der Einsatz von Kategorie2-Systemen könnte durch detaillierte Expositionserfassungen das Niveau des Experten-Screenings erweitern. Einfache messtechnische Verfahren können Untersuchende zukünftig auch auf Screening-Niveau durch objektive Analysen unterstützen und somit einen neuen Standard in der Gefährdungsbeurteilung physischer Belastungen ermöglichen.

\section{Fazit für die Praxis}

\section{- Eine Klassifizierung von kommer- zieller und nicht kommerzieller} Messtechnik sowie objektiver Bewertungsverfahren steht nun für die messdatenbasierte Gefährdungsbeurteilung physischer Belastungen der oberen Extremität zur Verfügung.

- Die Zuordnung der messtechnischen Lösungsansätze zu den Kategorien 1 bis 3 erfolgte unter anderem auf Basis von unterschiedlichen Nutzergruppen.

- Der Einsatz messdatenbasierter Gefährdungsbeurteilungen wird in Ergänzung zum bisherigen Vorgehen empfohlen.

- Die Weiterentwicklung von Sensorik inklusive Software zur Bewertung der Expositionsdaten ist zu befürworten, um zukünftig betrieblichen Praktikern den Einsatz der messdatenbasierten Gefährdungsbeurteilung bzw. Zugriff auf Messdaten zu ermöglichen.

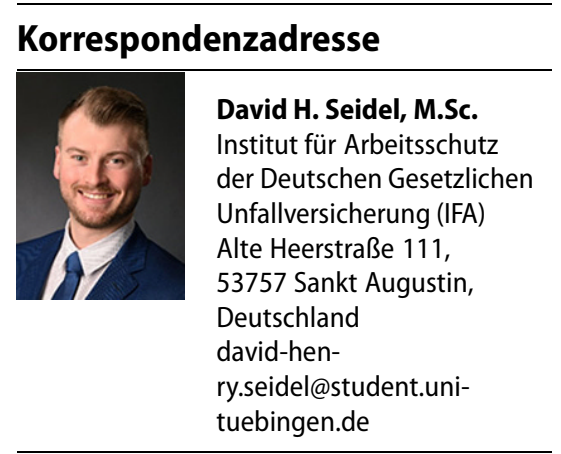

Förderung. Das Institut für Arbeitsmedizin, Sozialmedizin und Versorgungsforschung, Universitätsklinikum Tübingen, erhält eine institutionelle Förderung durch den Verband der Metall- und Elektroindustrie Baden-Württemberg e. V. (Südwestmetall).

Author contribution. D.H. Seidel entwarf das Manuskript. D.H. Seidel, R.P. Ellegast und B. Weber entwickelten $-\mathrm{Abb}$. 1, 2 und 3. Alle Autorinnen und Autoren kommentierten den Entwurf des Manuskripts, das danach von D.H. Seidel überarbeitet wurde. Alle Autorinnen und Autoren stimmten der Endfassung des Manuskripts zu. Dieser Artikel ist auch Teil des Promotionsvorhabens des Erstautors (D.H. Seidel).

Funding. Open Access funding enabled and organized by Project DEAL.

\section{Einhaltung ethischer Richtlinien}

Interessenkonflikt. D.H. Seidel, R.P. Ellegast, M.A. Rieger, B. Steinhilber und B. Weber geben an, dass kein Interessenkonflikt besteht.

Für diesen Beitrag wurden von den Autoren keine Studien an Menschen oder Tieren durchgeführt. Für die aufgeführten Studien gelten die jeweils dort angegebenen ethischen Richtlinien.

Open Access. Dieser Artikel wird unter der Creative Commons Namensnennung 4.0 International Lizenz veröffentlicht, welche die Nutzung, Vervielfältigung, Bearbeitung, Verbreitung und Wiedergabe in jeglichem Medium und Format erlaubt, sofern Sie den/die ursprünglichen Autor(en) und die Quelle ordnungsgemäßnennen, einen Link zur Creative Commons Lizenz beifügen und angeben, ob Änderungen vorgenommen wurden.

Die in diesem Artikel enthaltenen Bilder und sonstiges Drittmaterial unterliegen ebenfalls der genannten Creative Commons Lizenz, sofern sich aus der Abbildungslegende nichts anderes ergibt. Sofern das betreffende Material nicht unter der genannten Creative Commons Lizenz steht und die betreffende Handlung nicht nach gesetzlichen Vorschriften erlaubt ist, ist für die oben aufgeführten Weiterverwendungen des Materials die Einwilligung des jeweiligen Rechteinhabers einzuholen.

Weitere Details zur Lizenz entnehmen Sie bitte der Lizenzinformation auf http://creativecommons.org/ licenses/by/4.0/deed.de.

\section{Literatur}

1. Akkas O, Azari DP, Chen C-HE et al (2015) A hand speed and duty cycle equation for estimating the ACGIH hand activity level rating. Ergonomics 58:184-194

2. Álvarez D, Alvarez JC, González RC et al (2016) Upper limbjoint angle measurement in occupational health. Comput Methods Biomech Biomed Engin 19:159-170

3. Arbschg (1996) Gesetz über die Durchführung von Maßnahmen des Arbeitsschutzes zur Verbesserung der Sicherheit und des Gesundheitsschutzes der Beschäftigten bei der Arbeit. „Arbeitsschutzgesetz (ArbSchG) vom 7. August 1996 (BGBI.IS. 1246), das zuletzt durch Artikel 427 der Verordnung vom 31. August 2015 (BGBI. I S. 1474) geändert worden ist". Bundesministerium der Justiz und für Verbraucherschutz, Bundesamt für Justiz, S1-11

4. Balogh I, Arvidsson I, Björk J et al (2019) Work-related neck and upper limb disorders - quantitative exposure-response relationships adjusted for personal characteristics and psychosocial conditions. BMC Musculoskelet Disord 20(139):1-19

5. Barrero LH, Pulido JA, Berrio S et al (2012) Physical workloads of the upper-extremity among workers of the Colombian flower industry. Am J Ind Med 55:926-939

6. BAuA (2019) MEGAPHYS - Mehrstufige Gefährdungsanalyse physischer Belastungen am Arbeitsplatz. Gemeinsamer Abschlussbericht der BAuA und der DGUV Bd. 1. Bundesanstalt für Arbeitsschutz und Arbeitsmedizin (BAuA), Dortmund, Berlin, Dresden, S1-987

7. Bobin M, Amroun H, Coquillart S et al (2017) DNN based approach for the assessment of elbow flexion with smart textile sensor. In: SMC 2017-IEEE international conference on systems, man, and cybernetics Banff (hal-01677679v2)

8. Boudet G, Chausse P, Thivel D et al (2019) How to measure sedentary behavior at work? Front. Public Health 7:1-11

9. DGUV (2020) MEGAPHYS: Mehrstufige Gefährdungsanalyse physischer Belastungen am Arbeitsplatz (DGUV Report 3/2020). Abschlussbericht zum Kooperationsprojekt von BAuA und DGUV Bd. 2. Deutsche Gesetzliche Unfallversicherung e. V. (DGUV), Berlin, S1-984

10. Ditchen D, Ellegast RP, Rehme G (2010) GonKatast - Ein Messwertkataster zu beruflichen Kniebelastungen (IFA-Report 1/2010). Institut für Arbeitsschutz der Deutschen Gesetzlichen Unfallversicherung (IFA), Sankt Augustin, S1-137

11. Ellegast RP (2010) Quantifizierung physischer Belastungen am Arbeitsplatz. Zbl Arbeitsmed 60:386-389

12. Eu-Osha (2020) Gesunde Arbeitsplätze - entlasten Dich! Leitfaden zur Kampagne. Europäische Agentur für Sicherheit und Gesundheitsschutz am Arbeitsplatz (EU-OSHA). Eu-Osha, Luxemburg, S $1-36$

13. Forsman $M$ (2016) Ergonomic risk assessments-a need for reliable and attractive methods. In: Järvelin-Pasanen S (Hrsg) NES2016-Ergonomics in theory and practice-48th Annual Conference of Nordic Ergonomics and Human Factors Society University Eastern Finland, Kuopio. Report and Studies in Health Sciences, S 28-32

14. Grooten W, Johanssons E (2018) Observational methods for assessing ergonomic risks for workrelated musculoskeletal disorders. A scoping review. Rev Cienc Salud 16(especial):8-38

15. Hartmann B, SpallekM, Ellegast R(2013) Arbeitsbezogene Muskel-Skelett-Erkrankungen. Ursachen - 
Prävention - Ergonomie - Rehabilitation. ecomed MEDIZIN, Zwickau, S1-440

16. Holtermann A, Mathiassen SE, Pinder A et al (2017) Assessing sedentary behaviour at work with technical assessment systems-final report. Partnership for European Research in Occupational Safety and Health (PEROSH), S 1-53

17. Holtermann A, Schellewald V, Mathiassen SE et al (2017) A practical guidance for assessments of sedentary behavior at work: a PEROSH initiative. ApplErgon 63:41-52

18. Lin J-H, Kirlik A, Xu X (2018) New technologies in human factors and ergonomics research and practice. ApplErgon 66:179-181

19. Merino G, Da Silva L, Mattos D et al (2019) Ergonomic evaluation of the musculoskeletal risks in a banana harvesting activity through qualitative and quantitative measures, with emphasis on motion capture (Xsens) and EMG. Int J Ind Ergon 69:80-89

20. Nordander C, Ohlsson K, Åkesson I et al (2013) Exposure-response relationships in work-related musculoskeletal disordersinelbows and hands-a synthesis of group-level data on exposure and response obtained using uniform methods of data collection. Appl Ergon 44:241-253

21. Peppoloni L, Filippeschi A, Ruffaldi E et al (2016) A novel wearable system for the online assessment of risk for biomechanical load in repetitive efforts. Int JInd Ergon 52:1-11

22. Ranavolo A, Draicchio F, Varrecchia T et al (2018) Wearable monitoring devices for biomechanical risk assessment at work: current status and future challenges-a systematic review. Int J Environ Res Public Health 15:1-26

23. Rhén IM, Forsman M (2020) Inter- and intrarater reliability of the OCRA checklist method in video-recorded manual work tasks. Appl Ergon 84:103025

24. Schneider E, Irastorza X, Copsey S (2010) OSH in figures: work-related musculoskeletal disorders in the EU-facts and figures. In: European Agency for Safety and Health at Work (EU-OSHA) (Hrsg) European risk observatory report. Publications Office of the European Union, Luxembourg, $\mathrm{S}$ $1-184$

25. Seidel DH, Ditchen DM, Hoehne-Hückstädt UM et al (2019) Quantitative measures of physical riskfactors associated with work-related musculoskeletal disorders of the elbow: a systematic review. Int J Environ Res Public Health 16:1-23

26. Seidel $\mathrm{DH}$, Heinrich $\mathrm{K}$, Hermanns-Truxius I et a (2021) Assessment of work-related hand and elbow workloads using measurement-based TLV for HAL. ApplErgon 92:103310

27. Vignais N, Bernard F, Touvenot G et al (2017) Physical risk factors identification based on body sensor network combined to videotaping. Appl Ergon 65:410-417

28. Walmsley CP, Williams SA, Grisbrook T et al (2018) Measurement of upper limb range of motion using wearable sensors: a systematic review. Sports Med Open 4(53):1-22

29. Weber B, Douwes M, Forsman M et al (2018) Assessing arm elevation at work with technical assessment systems. Partnership for European Research in Occupational Safety and Health (PEROSH), S 1-49

30. Yang L, Grooten WJA, Forsman M (2017) An iPhone application for upper arm posture and movement measurements. Appl Ergon 65:492-500 